\title{
Freezing temperatures in the vaccine cold chain: A systematic literature review
}

\author{
Dipika M. Matthias ${ }^{\mathrm{a}, *}$, Joanie Robertson ${ }^{\mathrm{a}, 1}$, Michelle M. Garrison ${ }^{\mathrm{b}, 2}$, \\ Sophie Newland ${ }^{\mathrm{a}, 1}$, Carib Nelson ${ }^{\mathrm{a}, 1}$ \\ a PATH, 1455 NW Leary Way, Seattle, WA 98107, USA \\ ${ }^{\mathrm{b}}$ Child Health Institute - University of Washington Box 3564920 Seattle, WA 98195, USA
}

Received 19 December 2006; received in revised form 7 February 2007; accepted 16 February 2007

Available online 7 March 2007

\begin{abstract}
The dangers of accidental freezing of vaccines in the cold chain have prompted studies throughout the globe to better characterize the risk. To date, there has been no systematic review of these studies. This analysis highlights that accidental freezing is pervasive and occurs across all segments of the cold chain. Between $14 \%$ and $35 \%$ of refrigerators or transport shipments were found to have exposed vaccine to freezing temperatures, while in studies that examined all segments of distribution, between $75 \%$ and $100 \%$ of the vaccine shipments were exposed. More rigorous study designs were associated with higher levels of freeze exposure. As more expensive, freeze-sensitive vaccines are introduced into immunization schedules, freeze prevention will become increasingly critical for ensuring that the world's children are receiving fully potent vaccine.
\end{abstract}

(C) 2007 Elsevier Ltd. All rights reserved.

Keywords: Freeze-sensitive vaccines; Accidental freezing; Cold chain

\section{Introduction}

Vaccines are powerful public health tools that save an estimated 3 million lives each year [1]. Recognizing their importance, the global health community has prioritized the increased availability of vaccines to all the world's children. Yet this commitment has also had the effect of placing additional stress on an already fragile cold chain, the distribution network of equipment and procedures used to maintain vaccine quality from the vaccine manufacturer to the vaccine recipient.

\footnotetext{
* Corresponding author. Tel.: +1 206285 3500; fax: +1 2062856619.

E-mail addresses: dmatthias@ path.org (D.M. Matthias), jrobertson@path.org (J. Robertson), garrison@u.washington.edu (M.M. Garrison), snewland@path.org (S. Newland), cnelson@path.org (C. Nelson).

${ }^{1}$ Tel.: +1 206285 3500; fax: +1 2062856619 .

2 Tel.: +1 206616 1203; fax: +1 2066164623 .
}

World Health Organization (WHO) guidelines and manufacturer product inserts recommend that all vaccines except oral polio vaccine be kept at $2-8^{\circ} \mathrm{C}$ during in-country distribution. However, a poorly functioning cold chain may deviate from this target range and expose vaccines to freezing temperatures. Damage from accidental freezing can result in potency loss for freeze-sensitive vaccines such as diphtheria, tetanus, pertussis, liquid Haemophilus influenzae type b (Hib), hepatitis B, and inactivated polio virus [2-6]. However, cold chain practices tend to prioritize protecting vaccine from heat damage, often at the risk of exposure to freezing temperatures. As a result, accidental freezing of vaccines is a largely overlooked problem, yet freeze-sensitive vaccines represented over $31 \%$ of the US\$ 439 million UNICEF spent on all vaccines in 2005 [7].

Increased awareness of this danger has prompted studies of the cold chain, designed to characterize the risks of vaccine exposure to freezing temperatures. However, to date there has been no systematic review or cross-comparison of these 
studies. This paper attempts to review and analyze the current global data on freezing temperatures within the vaccine cold chain. The objective is to identify the settings in which freezing occurs and explore common factors among these studies and settings. It is our hope that this review will help raise further awareness of the extent to which vaccines are being exposed to freezing temperatures worldwide as well as encourage immunization program managers to design studies to investigate the state of their own cold chains and institute programmatic actions to prevent potential freeze exposure.

\section{Methods}

\subsection{Identification of cold chain studies}

To capture the broadest range of cold chain studies for inclusion within our analysis, a search for studies published between January 1985 and June 2006 was performed using four major electronic databases: PubMed, Popline, Embase, and Biosis. The term "vaccine" coupled with one of the following terms was searched in all databases: thermostability, stability, refrigerator, cold chain, storage, and temperature. In addition, we searched several public health websites for unpublished studies, including WHO, TechNet21, Epivac, and BASICS II. Finally, we solicited unpublished studies from the immunization community through a posting on the TechNet21 listserv. A total of more than 1200 articles were identified; the majority of articles were excluded based on subject matter inferred by the titles, while 117 warranted further review against our established inclusion criteria:

- Studies collecting primary data on the temperature conditions within existing cold chain infrastructures (during either transport or storage), using consistent procedures across the study.

- Studies using temperature-measuring devices that register freezing temperatures.

The resulting 35 articles that met the inclusion criteria are listed in Table 1. Of the 35 articles, 25 were published, while 10 were unpublished. Additionally, 3 of the 35 articles were from non-English sources.

\subsection{Data extraction methods}

For each of the studies that met the inclusion criteria, the following information was compiled: the number of samples measured (number of refrigerators or shipments), the percentage of samples that registered temperatures that were too cold, the temperature that was used as the cutoff for defining "too cold" in each study (freeze-exposure threshold), the period and frequency of temperature measurement, the type of measuring equipment used, the country in which the study was conducted, and the year in which the study was published or reported. From articles in which both transport and storage temperature data were collected, information about each segment was noted separately. Thus, some studies provided multiple data points.

\subsection{Design of analysis}

Transport and storage data were analyzed separately to accommodate for differences in endpoints across the various studies and also because the factors that could potentially influence temperature variations could be expected to differ between transport and storage situations. The data were also categorized as developed-country versus developing-country studies to facilitate comparisons across presumed differences in cold chain infrastructure [8]. To complement descriptive statistics, a series of multivariate meta-regression analyses was pursued to identify factors that might be associated with the level of exposure to freezing temperatures found in either transport or storage studies.

\subsection{Study limitations}

To date, a substantial proportion of the worldwide studies of freezing temperatures in the cold chain have been conducted in the Asia-Pacific region, with this bias reflected in the study set ( 13 out of the 35 studies included in the analysis). Furthermore, almost half of the developed-country studies were conducted in Australia ( 7 out of 16 studies). Therefore, although the study findings may be indicative, they prevent full characterization of the study outcomes as representative of the worldwide problem.

In addition to geographic clustering, publication bias the potential for studies with only certain types of results to be published - could also prevent the study results from being generalized. For this reason, much effort went into finding unpublished studies and resulted in $28 \%$ of the study set (10 out of 35 articles) coming from unpublished sources. A large percentage of unpublished sources may also better reflect the likely universe of studies on this topic, as vaccine freeze-exposure studies are more often conducted internally to guide systems improvements. Still, the findings from this study may not be generalized for all subcategories of analysis. For instance, we were only able to find two transport studies in developed countries, making it difficult to draw conclusions about vaccine temperature exposure under that particular scenario $[9,10]$.

\section{Results}

\subsection{Identified studies}

Of the 35 articles selected, 17 articles reported on research that was conducted in developed countries and 18 in developing countries. Each subset was then further divided into studies evaluating the temperatures of storage versus transport segments of the cold chain (Table 1). Two articles were dropped prior to the comparative analysis due to an inabil- 
Table 1

Cold chain studies evaluating vaccine storage or transport temperatures below the recommended range

\begin{tabular}{|c|c|c|c|c|c|c|}
\hline & Authors & Year & Country & Unit of analysis & $\begin{array}{l}\text { Sample } \\
\text { size }\end{array}$ & $\begin{array}{l}\text { Occurrence of temperatures } \\
\text { below freeze threshold }(\%)\end{array}$ \\
\hline 1 & Ford and Gibbs [23] & 1990 & New Zealand & Refrigerator & 27 & 26 \\
\hline 2 & Lugosi and Battersby [24] & 1990 & Hungary & Transport shipment & 166 & 38 \\
\hline 3 & Thakker and Woods [25] & 1992 & Great Britain & Refrigerator & 8 & 50 \\
\hline 4 & Bass [26] & 1993 & Papua New Guinea & Refrigerator & 17 & 6 \\
\hline 5 & Cheriyan [9] & 1993 & UK & Transport shipment & 7 & 0 \\
\hline 6 & Miller and Harris ${ }^{\mathrm{a}}[27]$ & 1994 & Australia & Vials & 59 & 48 \\
\hline 7 & Woodyard et al. [28] & 1995 & USA & Refrigerator & 27 & 26 \\
\hline 8 & Yuan et al. [29] & 1995 & Canada & Refrigerator & 135 & 6 \\
\hline 9 & Finnegan and Howell [30] & 1996 & Ireland & Refrigerator & 111 & 7 \\
\hline 10 & Hanjeet et al. $^{\mathrm{a}}[31]$ & 1996 & Malaysia & Vials & 234 & 99 \\
\hline 11 & Jeremijenko et al. [32] & 1996 & Australia & Refrigerator & 50 & 34 \\
\hline 12 & Guthridge and Miller [10] & 1997 & Australia & Transport shipment & 5 & 40 \\
\hline 13 & Kone [33] & 1997 & Mauritania & Refrigerator & 108 & 4 \\
\hline 14 & Steinglass [34] & 1997 & Kazakhstan & Refrigerator & 15 & 67 \\
\hline 15 & Wawryk et al. [35] & 1997 & Australia & Refrigerator & 40 & 53 \\
\hline 16 & Gold et al. [36] & 1998 & Australia & Refrigerator & 1 & 100 \\
\hline 17 & Reimer and Lewis [37] & 1998 & Australia & Refrigerator & 52 & 23 \\
\hline 18 & Gold et al. [38] & 1999 & Australia & Refrigerator & 32 & 22 \\
\hline 19 & Battersby and Feilden [39] & 2000 & Ukraine & Refrigerator & 1 & 100 \\
\hline 20 & Berhane and Demissie [40] & 2000 & Ethiopia & Refrigerator & 60 & 2 \\
\hline 21 & Bell et al. ${ }^{b}[41]$ & 2001 & USA & Refrigerator & 262 & 15 \\
\hline 22 & Lewis et al. [42] & 2001 & Australia & Refrigerator & 102 & 36 \\
\hline 23 & Munck [43] & 2001 & Kyrgyzstan & Refrigerator & 19 & 0 \\
\hline 24 & $\begin{array}{l}\text { TechNet } 21 \text { New Delhi } \\
\text { Consultation Report [44] }\end{array}$ & 2001 & Nepal & Refrigerator & 1 & 100 \\
\hline 25 & Gazmararian et al. [45] & 2002 & USA & Refrigerator & 641 & 7 \\
\hline 26 & Ortega Molina et al. [46] & 2002 & Spain & Refrigerator & 43 & 5 \\
\hline 27 & PATH [47] & 2002 & E. European Country & Refrigerator & 185 & 25 \\
\hline \multirow[t]{2}{*}{28} & PATH/University of & \multirow{2}{*}{2003} & \multirow{2}{*}{ Southeast Asian Country } & Refrigerator & 11 & 36 \\
\hline & Melbourne [11] & & & Transport shipment & 8 & 75 \\
\hline 29 & Edstam et al. [48] & 2004 & Mongolia & Transport shipment & 240 & 15 \\
\hline \multirow[t]{2}{*}{30} & \multirow{2}{*}{ Glele Kakaï [12] } & \multirow{2}{*}{2004} & \multirow{2}{*}{ Benin } & Refrigerator & 16 & 56 \\
\hline & & & & Transport shipment & 6 & 50 \\
\hline \multirow[t]{2}{*}{31} & \multirow{2}{*}{ Nelson et al. [13] } & \multirow{2}{*}{2004} & \multirow{2}{*}{ Indonesia } & Refrigerator & 14 & 29 \\
\hline & & & & Transport shipment & 16 & 44 \\
\hline \multirow[t]{2}{*}{32} & \multirow{2}{*}{ PATH, Village Reach [14] } & \multirow{2}{*}{2004} & South African & Refrigerator & 10 & 80 \\
\hline & & & Country & Transport shipment & 8 & 75 \\
\hline \multirow[t]{2}{*}{33} & \multirow{2}{*}{ PATH/UNICEF [15] } & \multirow{2}{*}{2005} & \multirow{2}{*}{ Bolivia } & Refrigerator & 25 & 60 \\
\hline & & & & Transport shipment & 11 & 100 \\
\hline 34 & McGuire [49] & 2006 & Kenya, Liberia, Pakistan & Transport shipment & 90 & 60 \\
\hline \multirow[t]{2}{*}{35} & \multirow{2}{*}{ Wirkas et al. [16] } & \multirow{2}{*}{2006} & \multirow{2}{*}{ Papua New Guinea } & Refrigerator & 16 & 31 \\
\hline & & & & Transport shipment & 12 & 100 \\
\hline
\end{tabular}

${ }^{a}$ These studies met our original inclusion criteria; however, the reported data points could not be compared on the same basis as the other studies. In the case of study \#6 and \#10, the percent of freeze-exposed vials could not be compared to freeze-exposed refrigerators or transport shipments since some of the vials may have been stored together, thereby obscuring the independence of the reported data points. Therefore, these studies do not appear in the comparative analysis.

b Additional data was acquired through e-mail correspondence with the author to resolve questions about the reported data such as what temperatures were measured during transport versus storage legs of cold chain assessment [13,15] how many unacceptable temperature readings were in the freezing range[32] or how many refrigerators were included per physician practice [41].

ity to compare the reported data points on the same basis as the other studies (marked with an asterisk in Table 1). Six of the remaining 33 articles collected data on both transport and storage segments. These data were divided and grouped with their respective transport and storage study sub- sets, resulting in a total of 39 data points for the comparative analysis.

The majority of transport studies analyzed all transport segments of the cold chain, from central storage to clinics. Most of the storage studies examined the lower levels of the 
Table 2

Summary of studies

\begin{tabular}{|c|c|c|}
\hline Cold chain segment & Developing countries & Developed countries \\
\hline \multicolumn{3}{|l|}{ Transport } \\
\hline Number of studies & 9 & 2 \\
\hline Study sample size ${ }^{\mathrm{a}}$ (mean, standard deviation [S.D.]) & $61.9(86.2)$ & $6(1.4)$ \\
\hline Freeze threshold temperature $\left({ }^{\circ} \mathrm{C}\right)$ (mean, S.D.) & $-0.4(1.0)$ & $0(0)$ \\
\hline Year study conducted (mean, S.D.) & $2003(4.9)$ & $1995(2.1)$ \\
\hline Rigorous monitoring ${ }^{\mathrm{b}}$ (duration and frequency) & $78 \%$ & $50 \%$ \\
\hline$\%$ shipments found below freeze threshold weighted mean $(95 \% \mathrm{CI})$ & $35.3 \%(14.8-55.8 \%)$ & $16.7 \%(0-44.9 \%)$ \\
\hline \multicolumn{3}{|l|}{ Storage } \\
\hline Number of studies & 14 & 14 \\
\hline Study sample size ${ }^{\mathrm{a}}$ (mean, S.D.) & $35.6(51.3)$ & $113.6(168.3)$ \\
\hline Freeze threshold temperature $\left({ }^{\circ} \mathrm{C}\right)$ (mean, S.D.) & $0(0)$ & $0.4(1.0)$ \\
\hline Year study conducted (mean, S.D.) & $2002(4.1)$ & $1997(3.8)$ \\
\hline Rigorous monitoring ${ }^{\mathrm{b}}$ (duration and frequency) & $50 \%$ & $29 \%$ \\
\hline$\%$ refrigerators found below freeze threshold, weighted mean $(95 \% \mathrm{CI})$ & $21.9 \%(10.3-33.6 \%)$ & $13.5 \%(6.4-20.7 \%)$ \\
\hline
\end{tabular}

cold chain, focusing on clinic refrigerators. The size of the studies varied, ranging from 1 to over 600 refrigerators or from 5 to over 200 transport shipments. There were also differences in the definition of the freeze-exposure threshold. While the majority of studies used $0^{\circ} \mathrm{C}$ for this threshold, some considered only temperatures below $-3{ }^{\circ} \mathrm{C}$, while others deemed anything below $2{ }^{\circ} \mathrm{C}$ as too cold. In some cases, the definition also included a time element to determine an unacceptable level of exposure. Another notable variation in design across the studies was the frequency and duration of the temperature monitoring.

\subsection{Comparison of studies and outcomes}

Key study design parameters (sample size, freezeexposure threshold, year the study was conducted, and level of monitoring rigor) were compared across four study scenarios (developed versus developing country, transport versus storage). These figures are summarized in Table 2.

\section{Discussion}

\subsection{Analysis of key findings}

- During transport, the occurrence of freezing temperatures was found to be $16.7 \%$ in developed countries compared to $35.3 \%$ in developing countries. This difference was not significant, potentially indicating that the current transport practice common to all countries - vaccines placed with frozen ice packs inside of insulated carriers - is placing vaccines at risk, regardless of the resource setting in which it is conducted. Observations indicate that the WHO-recommended practice of "conditioning" ice packs (allowing them to begin melting before placing them in transport cold boxes) is not routinely followed. Alternatively, the lack of statistical significance could also be driven by the relatively small number of included studies for the transport analysis in developed countries.

- During storage, the occurrence of exposure to freezing temperatures was found to be $13.5 \%$ in developed countries versus $21.9 \%$ in developing countries. Again, this difference was not found to be statistically significant, underscoring the fact that vaccine exposure to freezing temperatures in the cold chain appears to be a truly global problem, occurring in resource-rich as well as resourcelimited settings.

- The monitoring rigor of the study was found to be a significant predictor of freezing temperatures, implying that studies designed to gauge freezing temperatures with more frequent or continuous monitoring were more likely to report occurrences of vaccine freezing. Studies in both developed and developing countries with less rigorous monitoring reported significantly fewer occurrences of vaccine exposure to freezing temperature than studies in developing countries with scheduled or continuous monitoring $(55 \%$ less $[p<0.001]$, and $40 \%$ less $[p=0.001]$, respectively). Another important finding was that among the studies with more rigorous monitoring, there was no significant difference between developed- and developingcountry status in the occurrence of vaccines exposed to freezing temperatures $([p=0.23])$.

- In both the transport and storage analyses, the larger studies had less exposure of vaccines to freezing temperatures. However, after controlling for monitoring rigor in the storage analysis, it appears that the size of the study makes no difference in the observed proportions of vaccine exposed to freezing temperatures $([p=0.18])$. In fact, monitoring rigor was the only variable that carried significance, implying that study methods matter more than location or study 
size in terms of the proportion of vaccine freeze exposure found.

- In the six studies that analyzed the exposure of vaccine shipments to freezing temperatures as they traveled through both shipment and storage segments of the cold chain from either national or regional stores all the way to health clinics, the findings were even more striking: between $75 \%$ and $100 \%$ of the vaccine shipments were exposed to freezing temperatures at least once during the distribution process [11-16]. These comprehensive studies suggest that the risk of damaging freeze-sensitive vaccines is present in virtually every stage of the cold chain.

\subsection{Guidance for future studies}

Although this review cannot reach a conclusion about the proportion of vaccine that is actually damaged by exposure to freezing temperatures or actually results in moderated immunogenicity, we do know that potency loss does occur when freeze-sensitive vaccines undergo the phase change to a frozen state. Many epidemiological studies have also pointed to vaccine freezing as a possible contributor to low immune response in vaccinated individuals and the existing literature relating freeze exposure to potency loss is compelling enough to suggest some degree of impact on immune response [2-6,17-19]. More lab-based studies are needed to augment the literature base in order to gain a more precise understanding of the degree of potency loss associated with common field conditions. Detailed cold chain studies that monitor both time and temperature exposure across the entire length of the cold chain would provide the richest source of exposure conditions for such purposes.

It is also important to note that only 35 studies could be found in the global literature to date, highlighting the lack of attention to conditions which may be undermining immunization efforts worldwide. The sheer paucity of literature is a call to action for more rigorous and comprehensive studies that examine the exposure of vaccines to freezing temperatures through all transport and storage segments of the cold chain.

\subsection{Recommendations}

Freeze avoidance will become even more critical in the future as more expensive freeze-sensitive vaccines such as DTP-hepatitis B, liquid Hib, pneumococcal, and influenza are introduced into developing-country immunization programs. Fortunately, accidental exposure to freezing temperatures can be prevented. The following is a list of simple and proven tools available today that could help program managers minimize the potential for freeze damage to their vaccines:

- Investigate vaccine freezing in cold chains. WHO recommends that immunization programs use temperaturemonitoring studies to identify where problems are occurring so that corrective action can be taken. A WHO protocol has been developed and successfully applied in several countries [20,11,13-15].

- Apply innovative cold chain practices. Using cool water packs instead of frozen ice packs during cold box transport to avoid the freezing commonly associated with improper ice-pack conditioning [13], storing the more heat-stable vaccines in air-conditioned rooms, and conducting limited transport at ambient temperatures are possible freezeprevention approaches.

- Improve training. Recommended changes in training include educating vaccine managers and handlers about freeze-sensitive vaccines, providing clear guidelines on vaccine handling procedures, and increasing the focus on freeze prevention during supervision.

- Update cold chain infrastructure. Improved cold chain equipment is an important aspect of freeze prevention. New refrigerators are being developed that automatically control temperatures and eliminate the possibility of inadvertent vaccine freezing. At a minimum, $\min / \max$ thermometers can help users better understand the range of temperatures that vaccines are exposed to over time and can prompt mitigation strategies. Electronic temperaturemonitoring devices are also available to accompany vaccines during transport and storage, including low-cost devices that signal exposure to an established freezeexposure temperature [21].

- Take advantage of vaccines' heat stability. Freezing temperatures damage freeze-sensitive vaccines more quickly than ambient temperatures. Vaccine vial monitors, heatexposure indicators required on all vaccines distributed by UNICEF, allow vaccine programs to utilize the natural heat stability of some vaccines to expose them to temperatures warmer than $8^{\circ} \mathrm{C}$ for limited time without risk of heat damage. It may also be possible to allow a slight warming of the recommended cold chain temperatures to reduce freezing without causing heat damage [22].

- Create system-wide policy to avoid freeze damage. Policy changes (at the global and country level) are needed to prioritize the prevention of freezing in the cold chain and apply resources to raising awareness, training, and equipment infrastructure.

\section{Conclusion}

This analysis highlights that exposure of vaccines to freezing temperatures is pervasive, occurring in both developedand developing-country settings, as well as within both the storage and transport segments of the cold chain. Across the four scenarios analyzed, the average proportion of exposure ranges from $14 \%$ to $35 \%$. In the six studies that measured temperatures longitudinally through multiple sections of the cold chain, between $75 \%$ and $100 \%$ of the vaccine shipments were exposed to freezing temperatures. As vaccine availability expands and more expensive, freeze-sensitive vaccines are introduced into immunization programs, freeze preven- 
tion will become even more essential, both for making the most productive use of immunization dollars and for ensuring that the world's children are receiving fully potent and effective vaccines.

\section{Acknowledgements}

These materials were developed at PATH with support from the Bill \& Melinda Gates Foundation and the Bureau for Global Health, United States Agency for International Development (USAID), under the HealthTech Program, Cooperative Agreement \#GPH-A-00-01-0000500 . The opinions expressed herein are those of the authors and do not necessarily reflect the views of either the Bill \& Melinda Gates Foundation or USAID.

\section{References}

[1] UNICEF website. Available at: http://www.unicef.org/immunization/ index_coverage.html; accessed January 10, 2006.

[2] WHO. Temperature sensitivity of vaccines. Geneva: WHO; 2006. WHO/IVB/06.10.

[3] Boros CA, Hanlon M, Gold MS, Roberton DM. Storage at $-3{ }^{\circ} \mathrm{C}$ for $24 \mathrm{~h}$ alters the immunogenicity of pertussis vaccines. Vaccine 2001;19(25-26):3537-42.

[4] Ho MM, Mawas F, Bolgiano B, Lemercinier X, Crane DT, Huskisson $\mathrm{R}$, et al. Physico-chemical and immunological examination of the thermal stability of tetanus toxoid conjugate vaccines. Vaccine 2002;20(29-30):3509-22.

[5] Bolgiano B, Mawas F, Yost SE, Crane DT, Lemercinier X, Corbel MJ. Effect of physico-chemical modification on the immunogenicity of Haemophilus influenzae type b oligosaccharide-CRM(197) conjugate vaccines. Vaccine 2001;19(23-24):3189-200.

[6] Diminsky D, Moav N, Gorecki M, Barenholz Y. Physical, chemical and immunological stability of CHO-derived hepatitis B surface antigen (HBsAg) particles. Vaccine 1999;18(1-2):3-17.

[7] UNICEF Supply Division annual report page. UNICEF website. Available at: http://www.unicef.org/supply/index_report.html; accessed November 15, 2006.

[8] World Bank website. Available at: http://www.worldbank.org/data/ countryclass/countryclass.html; accessed July 2005

[9] Cheriyan E. Monitoring the vaccine cold chain. Arch Dis Childhood 1993;69(5):600-1.

[10] Guthridge SL, Miller NC. Cold chain in a hot climate. Aus NZ J Public Health 1997;20(6):657-60.

[11] PATH, University of Melbourne. Unpublished report; 2003.

[12] Glele Kakaï CF. Study of factors influencing the quality of EPI vaccines in the health district of Natitingou [Benin] during the period of January to June 2004. Thesis study, interuniversity program between Universite de Cocody, Abidjan, and University Paris Dauphine; November 2004.

[13] Nelson CM, Wibisono H, Purwanto H, Mansyur I, Moniaga V, Widjaya A. Hepatitis B vaccine freezing in the Indonesian cold chain: evidence and solutions. Bull World Health Organ 2004;82(2):99-105.

[14] Village Reach, PATH. Unpublished report; 2004.

[15] Nelson C, Froes P, Van Dyck AM, Chavarria J, Boda E, Coca A, et al. Monitoring temperatures in the vaccine cold chain in Bolivia. Vaccine 2007;25:433-7.

[16] Wirkas T, Toikilik S, Miller N, Morgan C, Clements CJ. A vaccine cold chain freezing study in PNG highlights technology needs for hot climate countries. Vaccine; August 31, 2006 [Epublish ahead of print].
[17] Edstam JS, Dulmaa N, Nymadawa P, Rinchin A, Khulan J, Kimball AM. Comparison of hepatitis B vaccine coverage and effectiveness among urban and rural Mongolian 2 year olds. Prev Med 2002;34(2):207-14.

[18] Klotz SA, Normand R, Silberman R. Hepatitis B vaccine in healthy hospital employees. Infect Control 1986;7(7):365-9.

[19] McLean AA, Shaw R. Hepatitis B vaccine. Ann Intern Med 1982;97(3):451.

[20] WHO. Study protocol for temperature monitoring in the vaccine cold chain. Geneva: WHO; 2005. WHO/IVB/05.01.

[21] WHO. Guidelines on the international packaging and shipping of vaccines. Geneva: WHO; 2005.

[22] Schondorf I, Banzhoff A, Nicolay U, Diaz-Mitoma F. Overcoming the need for a cold chain with conjugated meningococcal Group $C$ vaccine: a controlled, randomized, double-blind study in toddlers on the safety and immunogenicity of Menjugate(R), stored at room temperature for 6 months. Vaccine 2007;25:1175-82.

[23] Ford RPK, Gibbs CE. General practice storage of vaccines: a vital link in the cold chain. NZ Fam Phys 1990:178-81.

[24] Lugosi L, Battersby A. Transport and storage of vaccines in Hungary: the first cold chain monitor study in Europe. Bull World Health Organ 1990;68(4):431-9.

[25] Thakker Y, Woods S. Storage of vaccines in the community: weak link in the cold chain? BMJ 1992;304(6829):756-8.

[26] Bass AG. Vaccines in the national immunization programme. Papua New Guinea Med J 1993;36(2):141-57.

[27] Miller NC, Harris MF. Are childhood immunization programmes in Australia at risk? Investigation of the cold chain in the Northern Territory. Bull World Health Organ 1994;72(3):401-8.

[28] Woodyard E, Woodyard L, Alto WA. Vaccine storage in the physician's office: a community study. J Am Board Fam Practice 1995;8(2):91-4.

[29] Yuan L, Daniels S, Naus M, Brcic B. Vaccine storage and handling. Knowledge and practice in primary care physicians' offices. Can Fam Phys 1995;41:1169-76.

[30] Finnegan P, Howell F. Storage and handling of vaccines by family doctors. Irish Med J 1996;89(2):64-6, 68.

[31] Hanjeet K, Lye MS, Sinniah M, Schnur A. Evaluation of cold chain monitoring in Kelantan, Malaysia. Bull World Health Organ 1996;74(4):391-7.

[32] Jeremijenko A, Kelly H, Sibthorpe B, Attewell R. Improving vaccine storage in general practice refrigerators. BMJ 1996;312(7047):1651-2.

[33] Kone S. Draft report: evaluation of EPI and the cold chain in Mauritania. WHO; October 1997

[34] Steinglass data files, acquired by e-mail correspondence.

[35] Wawryk A, Mavromatis C, Gold M. Electronic monitoring of vaccine cold chain in a metropolitan area. BMJ 1997;315:518.

[36] Gold MS, Kemp AE, Osbourne M. Counting the cost of disrupting the vaccine cold chain. Med J Aust 1998;168(9):471-2.

[37] Reimer RF, Lewis PR. Vaccine storage in pharmacies on the Central Coast of New South Wales. Aust NZ J Public Health 1998;22:274-5.

[38] Gold MS, Martin L, Nayda CL, Kempe AE. Electronic temperature monitoring and feedback to correct adverse vaccine storage in general practice. Med J Aust 1999;171(2):83-4.

[39] Battersby A, Feilden R. Aspects of immunisation in Ukraine. Report from FBA Health Systems Analysts and CVP Consultants; September 2000.

[40] Berhane Y, Demissie M. Cold chain status at immunisation centres in Ethiopia. East Afr Med J 2000;77(9):476-9.

[41] Bell KN, Hogue CJ, Manning C, Kendal AP. Risk factors for improper vaccine storage and handling in private provider offices. Pediatrics 2001;107(6).

[42] Lewis P, Reimer R, Dixon A. Evaluating the efficacy of vaccine storage in general practice. Aust NZ J Public Health 2001;25(6):547-51.

[43] Munck MH. An assessment of the cold chain in Kyrgyzstan. WHO Consultation report; 2001.

[44] TechNet 21 New Delhi Consultation Report page. TechNet website. Available at: http://www.technet21.org/pdf_file/Delhireport.pdf; 2001 [accessed January 2006]. 
[45] Gazmararian JA, Oster NV, Green DC, Schuessler L, Howell K, Davis $\mathrm{J}$, et al. Vaccine storage practices in primary care physician offices: assessment and intervention. Am J Prev Med 2002;23(4):246-53.

[46] Ortega Molina P, Astasio Arbiza P, Albaladejo Vicente R, Gomez Rabago ML, de Juanes Pardo JR, Dominguez Rojas V. Vaccine storage cold chain at primary care centers in one area of Madrid: keeping the chain intact and degree of knowledge. Rev Esp Salud Publica 2002;76(4):333-46.
[47] Tyshchenko D, Romanyuk H, Mercer D. PATH. Unpublished report; 2002.

[48] Edstam JS, Dulmaa N, Tsendjav O, Dambasuren B, Densmaa B. Exposure of hepatitis $\mathrm{B}$ vaccine to freezing temperatures during transport to rural health centers in Mongolia. Prev Med 2004;39(2): 384-8.

[49] McGuire GA. Data from study of international shipments. Acquired by email correspondence. 